\title{
Caracterización de la Atención del Servicio de Urgencia Dental CESFAM Dr. Jorge Sabat, Valdivia.
}

\author{
Characterization of Dental Emergency Service CESFAM Dr. Jorge Sabat, \\ Valdivia.
}

\author{
Nicol Velásquez ${ }^{1 \mathrm{a}}$, Constanza Cárdenas $^{2 \mathrm{~b}}$ iD , Isabel Triviño ${ }^{2 \mathrm{c}}$, Pedro Aravena $^{2 \mathrm{~d}}$.
}

\section{RESUMEN}

Objetivo: Describir el perfil de la atención de urgencia odontológica ambulatoria de un Centro de Salud Familiar (CESFAM) de la ciudad de Valdivia.

Materiales y métodos: Estudio descriptivo realizado entre abril y julio 2019 en Servicio de Urgencia Dental CESFAM Dr. Jorge Sabat de Valdivia. Se registraron atenciones consecutivas a mayores de 18 años. Se obtuvo desde una ficha de recolección de datos, la zona de procedencia, historia de extracción de urgencia previa, nivel educacional, motivo de consulta y si correspondía, motivo de extracción. A través del formulario "Datos de Atención de Urgencias" se registró sexo, edad, diagnóstico, tratamiento y si correspondía, diente extraído.

Resultados: Se registraron 947 atenciones sin duplicado. En distribución por sexo, 53,8\% correspondió a femenino. El grupo etario más frecuente fue Adulto $(52,8 \%)$. La población urbana correspondió al 90,8\%. Lideró enseñanza media completa con $37,6 \%$. Mayor motivo de consulta fue el dolor (46,6\%). Los diagnósticos GES alcanzaron $37,7 \%$. La patología más prevalente fue periodontitis crónica $(35,9 \%)$ y el tratamiento más realizado fue exodoncia $(69,8 \%)$; los molares fueron los dientes más extraídos $(59,2 \%)$ y los usuarios con extracción de urgencia previa correspondieron al $62,7 \%$.

Conclusiones: Las características generales de la población revelan un déficit en salud oral, donde la mayor parte de los participantes consultan por dolor, en estados avanzados de las enfermedades, obteniendo principalmente extracciones dentales como tratamiento. En general, los resultados se condicen con estudios de salud nacionales y literatura científica previa, donde la demanda de atención es preferentemente de parte de adultos, mayormente mujeres, pertenecientes a zona urbana, con enseñanza media completa.
1. Centro de Salud Familiar Dr. Jorge Sabat Gozalo, Valdivia, Chile.

2. Universidad Austral de Chile, Valdivia, Chile.

a. Cirujana Dentista; Docente adjunto Universidad Austral.

b. Interna de sexto año Odontología

c. Cirujano-Dentista, práctica privada.

d. Cirujano-Dentista, Docente adjunto Universidad Austral, Doctor en Ciencias Médicas.

Correspondencia:

Constanza Cardenas

constanza.cardenas01@alumnos.uach.cl

PALABRAS CLAVES:

Servicios de Salud Dental, Emergencia, Atención Primaria de Salud, Salud Pública, Tratamiento de Urgencia, Chile.

\section{KEYWORDS :}

Dental Health Services, Emergency, Primary Health Care, Public Health, Emergency Treatment, Chile.

cC) () 2021 Applied Sciences in Dentistry - Universidad de Valparaíso, [Facultad de Odontología]. Este es un artículo Open Access bajo la licencia Atribución 4.0 Intemacional (CC BY 4.0) Creative Commons. (https://creativecommons.org/licenses/by/4.0/deed.es).

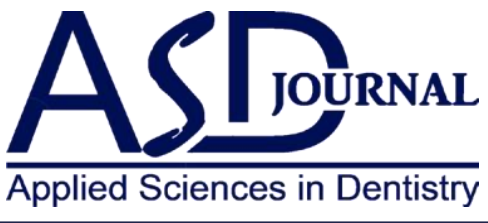




\section{ABSTRACT}

Objective: To describe the profile of the emergency ambulatory dental care in a Family Health Center (CESFAM) in the city of Valdivia.

Materials and methods: Descriptive study conducted between April and July 2019 in the Emergency Dental Service CESFAM Dr. Jorge Sabat of Valdivia. Consecutive care was recorded for those over 18 years of age. The following information was obtained from a data collection sheet: area of origin, history of previous emergency extraction, educational level, reason for consultation and, if applicable, reason for extraction. Through the "Emergency Care Data" form, sex, age, diagnosis, treatment and, if applicable, extracted tooth were recorded.

Results: 947 visits were recorded without duplication. In distribution by sex, $53.8 \%$ corresponded to women. The most frequent age group was adults (56.6\%). The urban population accounted for $90.8 \%$. It led the way in complete secondary education with $37.6 \%$. The greatest reason for consultation was pain (46.6\%). GES diagnoses reached 37.7\%. The most prevalent pathology was chronic periodontitis $(35.9 \%)$, and the most performed treatment was exodontia $(69.8 \%)$, . Molars were the most extracted teeth $(59.2 \%)$ and users with previous emergency extraction corresponded to $62.7 \%$.

Conclusions: The general characteristics of the population reveal a deficit in oral health, where most of the participants consult for pain, in advanced stages of the diseases, obtaining mainly dental extractions as treatment. In general, the results are in line with national health studies and previous scientific literature, where the demand for attention is preferably from adults, mostly women, belonging to urban areas, with complete secondary education.

\section{INTRODUCCIÓN}

La Salud Oral es una de las prioridades de salud en Chile, debido a la creciente percepción que tiene la población sobre ella y al impacto que tiene en la salud general y en la calidad de vida de los pacientes ${ }^{1,2}$. En este ámbito, la Urgencia Odontológica Ambulatoria, se define como un conjunto de patologías buco maxilo faciales, de aparición súbita, de etiología múltiple, que se manifiestan principalmente por dolor agudo y que provocan una demanda espontánea de atención ${ }^{3}$. En el año 2007, estas urgencias fueron incluidas en las Garantías Explícitas en Salud (GES), en busca de una solución al daño acumulado en la población adulta chilena y a la alta demanda existente ${ }^{5}$, convirtiéndose en la prestación GES más utilizada a nivel nacional ${ }^{6}$.

Estudios internacionales indican que las causas más frecuentes de consulta de urgencia dental son cuadros consecutivos a caries, enfermedades periodontales y trauma dentoalveolar ${ }^{2,7,8}$. Estudios nacionales muestran alta prevalencia de caries y enfermedad periodontal en la población general, sin embargo, no recogen información sobre la atención en el servicio de urgencias ni las características de los pacientes que requieren este tipo de atención ${ }^{9,10,11}$.

Un tratamiento entregado con frecuencia son las extracciones dentales, eliminando una parte vital para el funcionamiento del sistema estomatognático $^{12}$. En Chile se ha reportado alta prevalencia de edentulismo parcial o total $^{12,13}$, lo que puede generar graves consecuencias en términos de impacto social, psicológico y económico en las personas, las comunidades y los servicios de salud, además de conllevar grandes costes, incluyendo el costo financiero del tratamiento, el ausentismo escolar o laboral y la pérdida de ingresos que, a su vez, puede conducir a impactos en la comunidad en general por los gastos que deben asumir los servicios de salud para el tratamiento de la condición ${ }^{9}$. 
En los últimos años el sistema de urgencia odontológica del país ha sufrido modificaciones $^{5}$. Según la búsqueda realizada, existen escasos estudios a nivel nacional que analicen su situación actual, y los existentes se enfocan en evaluar satisfacción usuaria o la evolución de este sistema de manera retrospectiva $^{5,14}$. Por lo cual, el objetivo del presente estudio es describir el perfil de la atención de urgencia odontológica ambulatoria y sus usuarios en un Centro de Salud Familiar (CESFAM) de la ciudad de Valdivia.

\section{MATERIAL Y MÉTODOS}

Se realizó un estudio observacional descriptivo en el Servicio de Urgencia Dental del Centro de Salud Familiar Dr. Jorge Sabat Gozalo de la ciudad de Valdivia, durante el primer semestre del año 2019. Este estudio fue aprobado y autorizado por el Comité Ético Científico del Servicio de Salud de Valdivia (Ord. $\mathrm{N}^{\mathrm{o}}$ 076/2018, código de aprobación 1361470), el Departamento de Salud Municipal (DESAM) de la comuna de Valdivia y la Dirección del CESFAM. Se realizó un muestreo no probabilístico, por conveniencia a los usuarios del Servicio de Atención de Urgencias Dental del CESFAM Jorge Sabat que asistieron durante el primer semestre del año 2019, donde los invitados a participar firmaron un consentimiento informado. Se calculó el mínimo de atenciones a registrar a partir de las atenciones anuales en este servicio en el año 2018 (6948 atenciones), se estableció un nivel de confianza de $95 \%$, frecuencia esperada del $50 \%$ de pacientes que asistan por extracción dental y error aceptable del 3\%, arrojando un mínimo de 926 atenciones. Fueron incluidas en el estudio aquellas atenciones a usuarios mayores de 18 años, sin distinción por sexo, que firmaron el consentimiento informado, respondieron total y correctamente la encuesta y cuyos Datos de Atención de Urgencia (DAU) fueron indexados.

Fueron excluidas aquellas atenciones a usuarios con trastornos mentales que incapaciten dar su consentimiento, que no comprendían el idioma español o que consultaron más de una vez por el mismo diente y motivo de consulta.
En el caso de atención de usuarios analfabetos, tanto la encuesta como el consentimiento informado, fueron leídos en voz alta para su comprensión y en presencia de un testigo.

La recolección de datos se llevó a cabo a partir del formulario DAU completado digitalmente por el cirujano dentista de turno, además el paciente debió contestar una encuesta diseñada para el estudio, la cual fue aplicada por dos investigadoras. Desde el formulario DAU se extrajeron las variables: sexo (femenino/masculino), edad, rango etario (Jóvenes/ Adultos/ Adultos mayores), diagnóstico según Guía de atención de Urgencias Odontológicas y tratamiento. En los casos en los que el tratamiento de elección fue la extracción dental, se registró el tipo de diente (molar/ premolar/ canino/ incisivo). Desde la encuesta fueron extraídas las variables de: nivel educacional (sin educación básica, educación básica (completa/incompleta), educación media (completa/incompleta), educación superior (completa/incompleta)), zona de procedencia (rural/urbana), motivo de consulta (dolor, inflamación, trauma dento alveolar, desalojo restauración, extracción, movilidad) y extracción dental de urgencia previa (sí/no). En las atenciones donde el tratamiento fue la extracción, se registró el motivo de extracción (dolor/ motivo económico/ falta de tiempo para realizar tratamiento restaurador/ dudas sobre el pronóstico del tratamiento restaurador/ diente con indicación de extracción). Al momento de contestar la encuesta, se le explicó al paciente que debía señalar el motivo que sea más representativo o predominante respecto a su caso.

Para fines de este estudio, se consideró diente con indicación de extracción aquel que presente cualquiera de las siguientes condiciones: diente sin pronóstico rehabilitador, enfermedad periodontal con pérdida de inserción severa ${ }^{25}$, indicación protésica u ortodóncica. En el caso de los rangos etarios, se utilizó la clasificación del Instituto Nacional de Estadística (INE), el cual clasifica a la población Chilena según edad en 
Niños (0 a 14 años), Jóvenes (15 a 29 años), Adultos (30 a 59 años) y Adultos mayores (65 años o más $)^{29}$. Para fines del estudio, se consideró como jóvenes a los pacientes que tengan entre 18 y 29 años con el fin de que legalmente puedan firmar el consentimiento informado sin necesidad de un tutor.

Todos los datos fueron tabulados en un formulario electrónico Google Forms (Google Inc. Mountain View, CA, USA) para su posterior análisis. Las variables fueron analizadas mediante estadística descriptiva de frecuencias, excepto la edad, donde se utilizó estadística descriptiva de medias $y$ de dispersión (STATA v.14.0. StataCorp. Texas,

\section{RESULTADOS}

Se registraron 947 atenciones en el período comprendido entre abril y julio del año 2019 .

En la distribución por sexo, 53,5\% ( $\mathrm{n}=507)$ correspondió a femenino. La edad promedio fue de 41,91土16.4 años (95\% IC: 40.87 42.96 años), siendo para el sexo femenino un promedio de 41.75 土16.41 años (95\% IC: 40.32 - 43.18 años) y para el masculino de 41.1 土16.41 años (95\% IC: 40.56 - 43.64 años). El detalle de la distribución por grupo etario, procedencia y nivel educacional se representan en la Tabla I (Tabla I).

\begin{tabular}{llllll} 
& & \multicolumn{2}{l}{ Femenino (N=507) } & \multicolumn{2}{c}{ Masculino (n=440) } \\
\hline Variable & & $\mathrm{n}$ & $\%$ & $\mathrm{n}$ & $\%$ \\
\hline Rango etario & Joven & 157 & 30.97 & 132 & 30 \\
& Adulto & 264 & 52.07 & 236 & 53.64 \\
& Adulto mayor & 86 & 16.96 & 72 & 16.36 \\
\hline Procedencia & Rural & 47 & 9.27 & 41 & 9.32 \\
& Urbano & 460 & 90.73 & 399 & 90.68 \\
\hline Nivel educacional & Educación básica completa & 53 & 10.45 & 40 & 9.09 \\
& Educación básica incompleta & 37 & 7.3 & 48 & 10.91 \\
& Educación media completa & 196 & 38.66 & 163 & 37.05 \\
& Educación media incompleta & 94 & 18.54 & 76 & 17.27 \\
& Educación superior completa & 63 & 12.43 & 57 & 12.95 \\
& Educación superior incompleta & 62 & 12.23 & 51 & 11.59 \\
& Sin educación básica & 2 & 0.39 & 5 & 1.14 \\
\hline
\end{tabular}

Tabla I: Distribución de datos sociodemográficos según sexo $(n=947)$ 


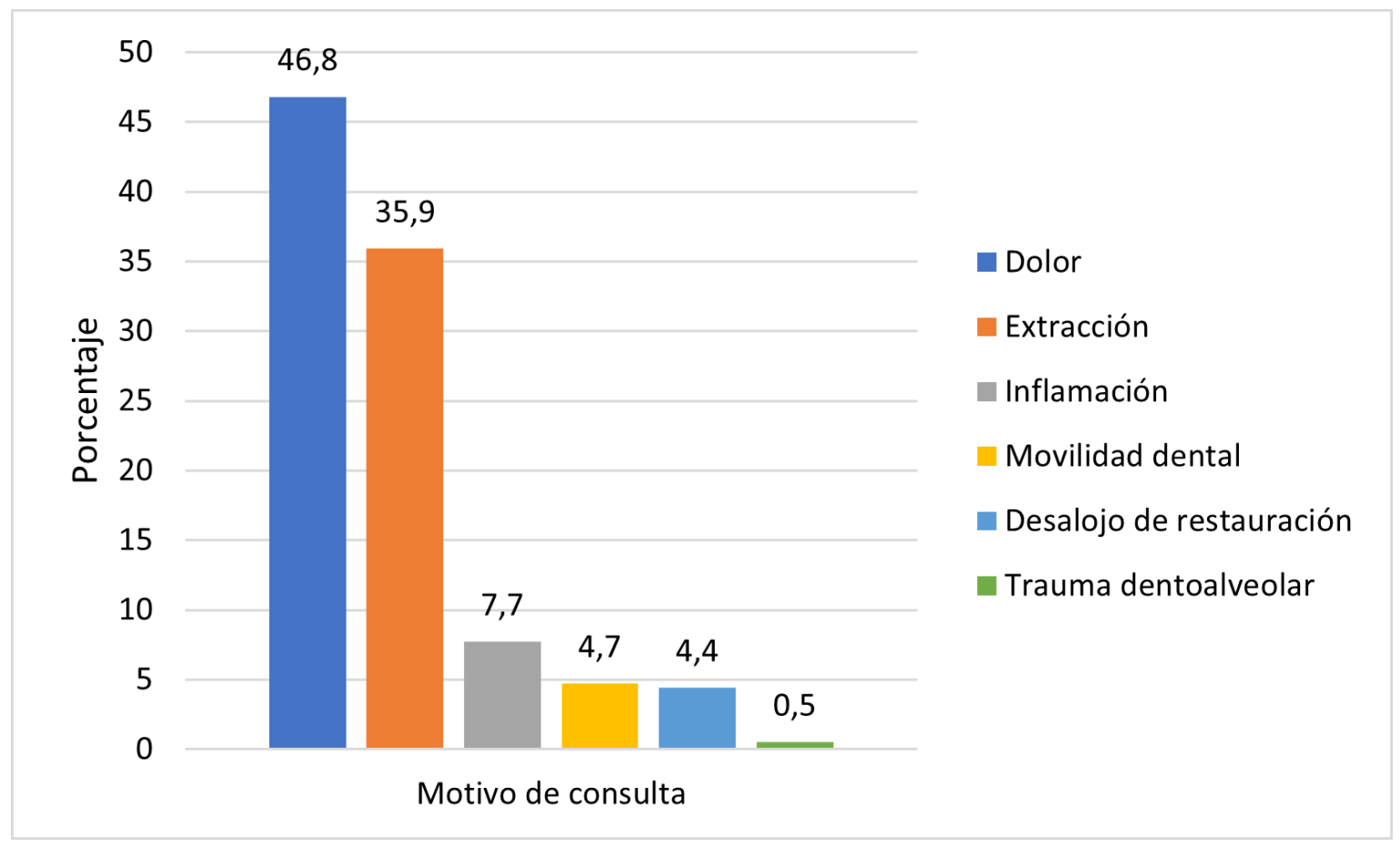

Figura I: Distribución porcentual de la población según motivo de consulta ( $n=947)$

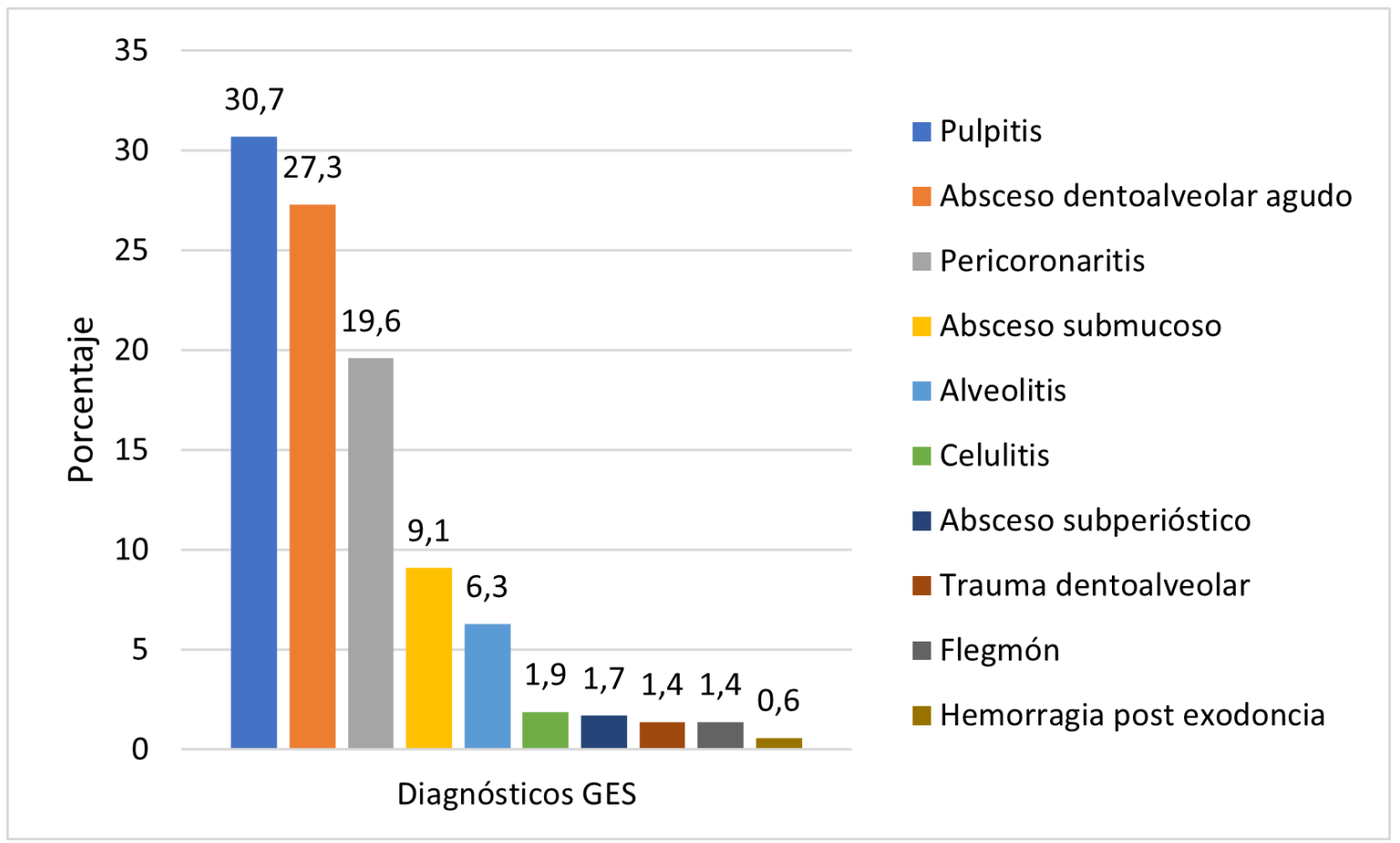

Figura II: Distribución porcentual de la población según diagnósticos GES ( $n=362)$ 


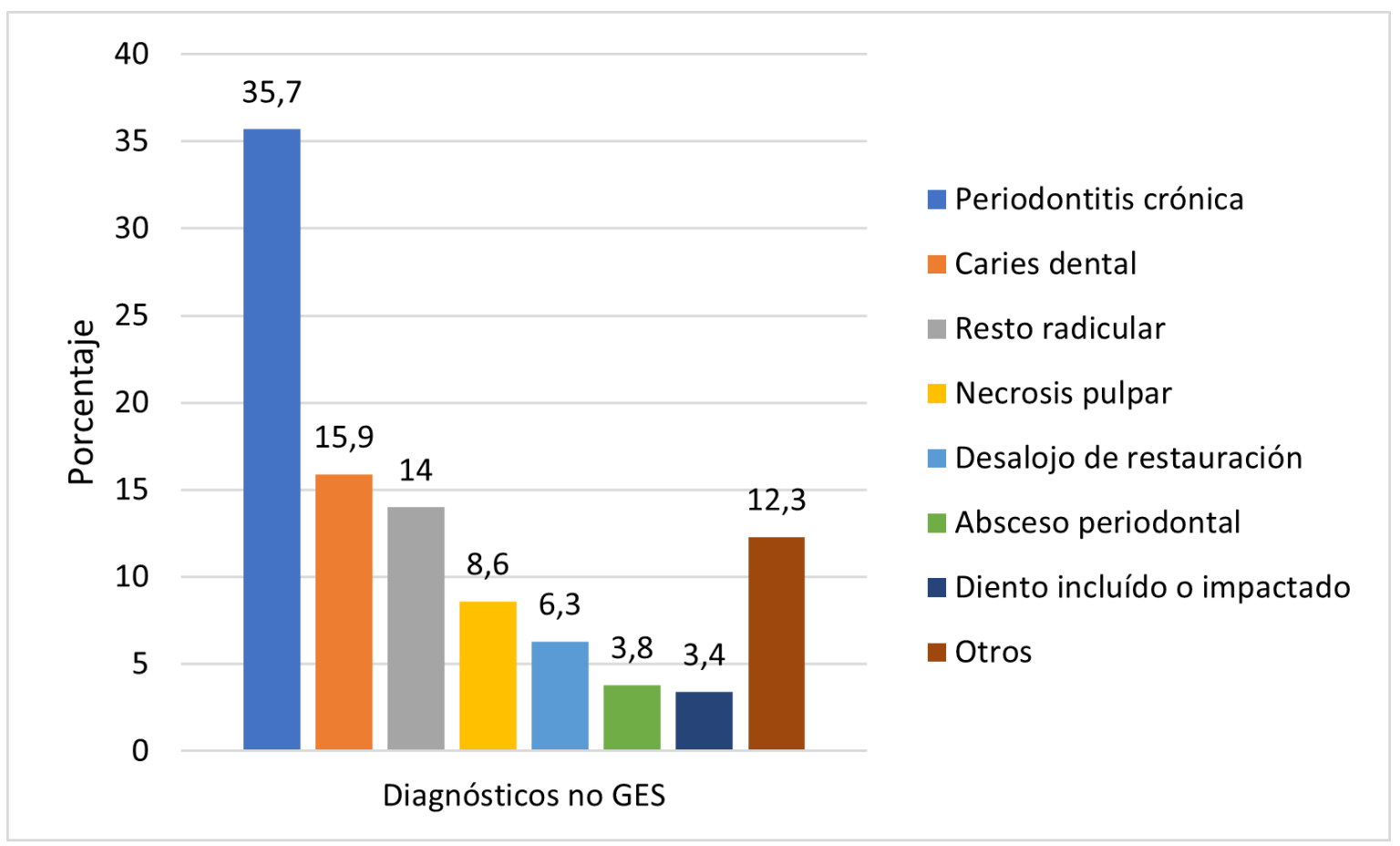

Figura III: Distribución porcentual de la población según diagnósticos no GES. $(n=585)$

$$
\text { Femenino }(\mathrm{N}=507) \quad \text { Masculino }(\mathrm{n}=440) \quad \text { Total }(\mathrm{n}=947)
$$

\begin{tabular}{lcccccc}
\hline & $\mathrm{n}$ & $\%$ & $\mathrm{n}$ & $\%$ & $\mathrm{n}$ & $\%$ \\
\hline Exodoncia & 326 & 64.3 & 317 & 72.05 & 643 & 67.9 \\
Derivación & 57 & 11.24 & 37 & 8.41 & 94 & 9.93 \\
Control & 34 & 6.71 & 34 & 7.73 & 68 & 7.18 \\
Tratamiento pericoronaritis & 28 & 5.52 & 6 & 1.36 & 34 & 3.59 \\
Restauración & 20 & 3.94 & 13 & 2.95 & 33 & 3.48 \\
Tratamiento Alveolitis & 12 & 2.37 & 11 & 2.5 & 23 & 2.43 \\
otros & 30 & 5.91 & 22 & 5 & 42 & 4.43 \\
\hline
\end{tabular}

Tabla II: Distribución de tratamientos realizados según sexo $(n=947)$ 
La distribución porcentual de atenciones según motivos de consulta se grafica en la figura 1 (Figura 1). Los diagnósticos pertenecientes a GES de urgencias correspondieron al 38,2 \% $(n=362)$ del total de las atenciones y los no GES alcanzaron una frecuencia de $61,8 \%$ $(n=585)$. La distribución porcentual de los diagnósticos GES y no GES, se grafica en la figura 2 y 3 respectivamente (Figura 2, 3).

El tratamiento más realizado fue la extracción dental con un $67,9 \% \quad(n=643)$, la distribución porcentual de los tratamientos realizados según sexo se encuentra graficada en la Tabla II (Tabla II). En el 62,3\% (n=590) de las atenciones, los usuarios ya habían recibido extracción de urgencia previamente.

En las atenciones en las que se realizó extracción dental, la indicación de extracción fue el motivo más prevalente con un $83,4 \%$ $(n=536)$; cuando ésta no estaba indicada, los usuarios decidían optar por la extracción por motivo económico en un $6,8 \%(\mathrm{n}=44)$ de los casos, por dolor en un $6,8 \%(n=44)$, por falta de tiempo para realizar el tratamiento ideal en un $2,2 \%(n=14)$ y por dudas en el pronóstico rehabilitador del diente en un $0,7 \%(n=5)$. El tipo de diente extraído con mayor frecuencia fueron molares con un $59,1 \%(n=396)$, seguido por premolares en un $25,2 \%(n=169)$, incisivos en un $11,1 \%(n=74)$ y en menor medida los caninos con un $4,6 \%(n=31)$.

\section{DISCUSIÓN}

Las características generales de la población estudiada revelan un déficit en la situación de salud oral, donde la mayor parte de los participantes consultan al odontólogo por dolor, en estados avanzados de las enfermedades, obteniendo principalmente extracciones dentales como tratamiento. Estas condiciones de salud oral podrían ser el resultado de la suma y resta de un verdadero mosaico de situaciones, como la falta de acceso a programas preventivos en edades más tempranas, creencias culturales, problemas para acceder al tratamiento ideal, entorno familiar, educación del paciente, entre otros ${ }^{12}$.
Según lo observado en la investigación, existe predominio del sexo femenino, concordando con la relación porcentual según sexo de la población validada por FONASA para el CESFAM Dr. Jorge Sabat, en el año 2018, donde el $54 \%$ de los usuarios inscritos corresponden al sexo femenino. Respecto a esto, cabe mencionar que el género cruza la mayoría de los otros determinantes de salud, en la medida en que éstos impactan a mujeres y hombres en forma diferenciada. En salud, el género determina desigualdades que se expresan en que las mujeres enferman más que los hombres durante todo el ciclo de vida ${ }^{28}$.

Respecto al rango de edad, los adultos entre 30 a 59 años son los que consultan con mayor frecuencia, resultado concordante con lo encontrado por Bucchi en 2012, en un estudio realizado en cinco establecimientos de atención primaria de salud en Chile, donde el 60,9\% de los usuarios del servicio de atención de urgencia odontológica correspondía a adultos entre 20 a 60 años ${ }^{14}$. Estos hallazgos podrían ser explicados por falta de acceso que tuvieron en edades más tempranas a programas preventivos, medidas de autocuidado y tratamientos curativos eficientes y oportunos ${ }^{15}$. Por otra parte, actualmente los esfuerzos se centran en prevenir y reducir la morbilidad bucal de mayor prevalencia en los menores de 20 años $^{1}$ y también se han focalizado programas específicos para adultos mayores ${ }^{15}$; buscando resolver el gran daño acumulado en adultos solo a través del programa de morbilidad odontológica.

Otro resultado de nuestro estudio indica que la población urbana consulta en mucho mayor medida que la población rural, esto está de acuerdo con lo expuesto en la Encuesta Nacional de Salud 2010- 2011, donde la muestra de personas cuya última visita al odontólogo ocurrió hace menos de 6 meses, es significativamente mayor en los participantes residentes en la zona urbana que en la zona rural $(24,7 \%$ y $16,2 \%$, respectivamente), mientras que el porcentaje que hace más de 5 años visitó al odontólogo es significativamente 
mayor en zona rural ${ }^{23}$. Esto podría indicar una barrera al acceso a la atención ${ }^{16}$, ya que la población rural se ve limitada por el horario de la atención, dada en su mayor parte en el horario nocturno, dificultando el traslado a su residencia.

Respecto al nivel educacional, la población estudiada presenta en su mayoría enseñanza media completa o superior. Este resultado es similar a lo encontrado por Bucchi, donde se muestra un $50,2 \%$ de la población con educación media completa o incompleta ${ }^{14}$. De la misma manera, Soto, en su estudio en Venezuela señala un 30,2 \% de la muestra con preuniversitario completo, seguida de técnico medio aprobado (26,2 \%), y nivel universitario $(22,2 \%)^{17}$. En este aspecto se destaca la importancia de la labor educativa de salud, la cual debe dirigirse a la población en general, independientemente del grado educacional e intelectual $^{26}$. También valorar la importancia de otros factores que pueden influir en el autocuidado, como creencias culturales, factores familiares o condición social, que pueden variar a lo largo de la vida ${ }^{27}$.

Por otra parte, el motivo de consulta predominante fue el dolor, según la literatura internacional, este es el más frecuente en la urgencia odontoestomatológica, tanto en los Servicios de Urgencias Extrahospitalarios, como Hospitalarios ${ }^{7}$ y requiere, en la mayoría de las ocasiones, atención profesional inmediata por la grave invalidez que ocasiona $^{18}$. Los datos epidemiológicos del dolor de origen dental varían dependiendo de la población estudiada, destacando la alta prevalencia que supone en la población general que oscila entre $19 \%$ y $66 \%{ }^{19}$. El motivo de consulta menos prevalente fue el trauma dentoalveolar $(0,5 \%)$, este hecho puede ser explicado por no incluir a la población infantil dentro del estudio, respecto a esto, según Fodor, en niños menores de 7 años, la consulta de mayor frecuencia es el trauma dentoalveolar, comúnmente causado por caídas $\mathrm{y}$ accidentes en bicicletas ${ }^{20}$.

Los diagnósticos calificados como urgencia
GES, fueron menos prevalentes que los diagnósticos no pertenecientes a GES. Esto podría estar dado porque las patologías incluidas en el GES de Atención de Urgencia Odontológica abarcan un número limitado de patologías, las cuales presentan sintomatología más aguda y dolorosa ${ }^{3}$.

La patología más prevalente en las atenciones fue la periodontitis crónica, este resultado está de acuerdo con lo encontrado en el primer estudio nacional de salud bucal, en adultos chilenos, donde se muestra un gran daño de las estructuras de soporte dentario, con la presencia de pérdida de inserción clínica $\geq 6$ $\mathrm{mm}$ en $58,3 \%$ de los adultos y $81,4 \%$ del grupo de 65 a 74 años ${ }^{9}$. En el estudio de casos y controles realizado por Quezada, se concluye que la reforma de salud a través del GES Salud Oral Integral de 60 años, demuestra variar parámetros clínicos de los pacientes beneficiarios, pero no logra mantener la salud periodontal ni el manejo de los factores de riesgo ${ }^{21}$. Esto puede explicarse por la ausencia de seguimiento y control para asegurar el éxito del tratamiento periodontal, en este aspecto este programa debe profundizar en estrategias efectivas para lograr cambios de conductas pues lo que se hace actualmente resulta insuficiente ${ }^{10}$.

La extracción dental fue el tratamiento más realizado y en la mayoría de las atenciones los usuarios ya habían recibido extracción de urgencia previa, siendo un porcentaje menor que al encontrado previamente en el estudio de Gamonal en Chile, donde el 87,3\% de los pacientes ya presentaba al menos una extracción por caries ${ }^{13}$. También es menor que lo encontrado por Carneiro en Brasil, donde el $87.7 \%$ de la muestra presentaba pérdida dentaria $^{22}$. Según Olate, en la selección de este tipo de tratamiento, sin duda alguna tiene una gran importancia la situación socioeconómica del paciente, donde debemos prestar especial atención a la población de escasos recursos y del ámbito rural, ya que, en la mayoría de los casos, presentan menos acceso a la atención odontológica $^{12}$. 
La extracción dental por indicación fue el motivo más prevalente, esto puede indicar poca efectividad de parte de programas preventivos y programas restaurativos que no intervienen oportunamente. Al igual que Monsalves, consideramos que uno de los determinantes más relevantes para mantener la salud oral en una población sana, o en una población tratada, es el control de salud, pues este nos permite pesquisar a tiempo factores de riesgo y lesiones de caries incipientes, pudiendo llegar a tiempo para intervenir prematuramente a nuestros pacientes con medidas más simples, con un enfoque menos invasivo y con un menor $\operatorname{costo}^{24}$.

De acuerdo con el estudio de Carneiro, la extracción dental se relaciona directamente con la enfermedad de caries, pero se deben considerar también otros gatillantes que llevan a la extracción como motivos económicos, culturales, la accesibilidad a programas de salud oral y la carencia de tiempo del usuario para asistir a la atención odontológica ${ }^{22}$, en este aspecto, en nuestro estudio, cuando la extracción no estaba indicada, los usuarios decidían optar por la extracción principalmente por motivo económico o dolor.

Carneiro en 2012 registra el motivo de extracción no indicado por el cirujano dentista como "voluntad propia", obteniendo un $17.96 \%$ de los casos y concluye que, en general, el paciente a menudo opta por la extracción, ya que se considera la solución más práctica y económica. Este resultado podría indicar que el código de ética profesional no se está cumpliendo adecuadamente, ya que corresponde al dentista llevar a cabo la motivación y la educación para la salud, desarrollando así su papel en la promoción de la salud ${ }^{22}$.

Dentro de las limitaciones de este estudio podemos mencionar las inherentes a la ficha de recolección de datos, donde las respuestas pueden ser afectadas por un sesgo de memoria del usuario al momento de consultar si anteriormente se había sometido a una extracción dental de urgencia, además de que el paciente puede entregar respuestas que crea socialmente más aceptadas. Se debe tener en cuenta, además, que esta ficha de recolección no ha sido validada. Por otra parte, cabe mencionar que este estudio fue llevado a cabo durante el primer semestre del año, por lo que, al realizar una recopilación de información durante un año completo, los resultados pueden variar.

En cuanto a las implicancias de los resultados y considerando las limitaciones presentadas, los servicios de atención primaria odontológica podrían considerar las características sociodemográficas encontradas, a la hora de confeccionar material de educación y desarrollar estrategias de intervención que permitan el control y disminución de los factores de riesgo asociados directamente a la aparición de urgencias estomatológicas. Por otra parte, se puede tener en cuenta lo expuesto a la hora de organizar los servicios, por ejemplo, para aumentar el número de atenciones disponibles para el servicio de periodoncia debido a su alta prevalencia. Este estudio podría además tomarse como una referencia inicial para realizar más estudios descriptivos a lo largo del país, donde se recomienda incluir un espectro más amplio de pacientes y de tiempo, para evaluar la necesidad de reorientar los servicios entregados a través de políticas públicas destinadas a promover la salud bucal y proveer mejores servicios para población.

\section{CONCLUSIONES}

Las características generales de la población estudiada revelan un déficit en la situación de salud oral, donde la mayor parte de los participantes consultan al odontólogo por dolor, en estados avanzados de las enfermedades, obteniendo principalmente extracciones dentales como tratamiento. En general, los resultados se condicen con los estudios de salud nacionales y literatura científica previa. Estas condiciones de salud oral podrían ser el resultado de distintas variables, como falta de acceso a programas preventivos en edades más tempranas, creencias culturales, dificultades para acceder 
al tratamiento ideal, entre otros.

\section{CONFLICTOS DE INTERÉS}

Sin conflictos de interés

\section{REFERENCIAS}

[1] Ministerio de Salud (MINSAL). Departamento de Salud Bucal. Plan Nacional de Salud Bucal 2018-2030. Santiago de Chile, Ministerio de Salud. 2017. p.6.

[2] Cigales A, Chaviano M, Sánchez Z, Robaina E, García M. Comportamiento epidemiológico de urgencia por caries dental. Rev Med Electrón. 2011; 33(4):416-422.

[3] Ministerio de Salud (MINSAL). Departamento de Salud Bucal. Guía Clínica de Urgencia Odontológica Ambulatorias. Santiago de Chile, Ministerio de Salud. 2011.

[4] Ministerio de Salud (MINSAL). Departamento de Salud Bucal. Garantías Explícitas en Salud- Salud bucal. Santiago de Chile, Ministerio de Salud. 2015.

[5] Olate V, Olate G, Cártes B, Olate S. Urgencias Odontológicas en la Provincia de Cautín - Chile, entre los Años 2009 a 2013. International journal of odontostomatology. 2014; $\quad$ 8(3): 447-52. DOI:10.4067/S0718381X2014000300020

[6] Fondo Nacional de Salud (FONASA). Oficio Cuenta Pública Fonasa. FONASA; Santiago de Chile. 2017.

[7] Currie C, Stone S, Durham J. Pain and problems: a prospective cross-sectional study of the impact of dental emergencies. J Oral Rehabil. 2015; 42(12):883-9.

DOI: 10.1111/joor.12333.

[8] Martínez D, Álvarez J, Clavera T, Montenegro Y. Caracterización de las urgencias estomatológicas atendidas en dos policlínicos docentes del municipio Playa. Rev haban cienc méd. 2015; 14(4): 488-95.

[9] Carvajal P. Enfermedades periodontales como un problema de salud pública: el desafío del nivel primario de atención en salud. Rev Clin Periodoncia Implantol Rehabil Oral. 2016; 9 (2): 177-83.

DOI: 10.1016/j.piro.2016.07.001

[10] Rojas C, Segovia J, Raccoursier V, Godoy J, Lopetegui M. Estado Periodontal y Necesidad de Tratamiento en Pacientes GES 60 Años de Villa Alemana. Rev. Clin. Periodoncia Implantol. Rehabil. Oral. 2010; 3(2):86-9. DOI:10.4067/S071901072010000200005.

[11] Ministerio de Salud (MINSAL). Departamento de Salud Bucal. Análisis de la situación de salud bucal en Chile. Santiago de Chile, Ministerio de Salud. 2010. p3.
[12] Olate S, Alister J, Soto M, Alveal R, Fuentes $J$ et al. Extracciones e indicaciones de extracciones dentales en población rural chilena de 11 a 30 años. Av. Odontoestomatol. 2006; 22(2), 119-24.

[13] Gamonal J. Prevalencia de enfermedades periodontales y de caries dental en la población de 35-44 y de 65-74 años de nivel socioeconómico bajo y medio-bajo de la provincia de Santiago, región metropolitana, y determinación de los recursos humanos necesarios para su tratamiento. Rev. Fac. Odontol. Univ. Chile. 1996;14(1):56-7. DOI: 10.1016/S0718-5391(09)70027-8.

[14] Bucchi C, Sepúlveda C, Monsalves M, Bustos L. Descripción de la Satisfacción Usuaria de Pacientes que Reciben Atención de Urgencia Dental en Cinco Establecimientos de Atención Primaria de Salud. Int J Odontostomat. 2012; 6(3):275-80.

DOI:10.4067/S0718381X2012000300005.

[15] Ministerio de Salud (MINSAL). Departamento de Salud Bucal. Norma Técnica de Urgencia Odontológica. Santiago de Chile, Ministerio de Salud. 2003.

[16] Rubiños E, Rodríguez L, Varela A, Varela $F$, Blanco A et al. Caracterización del usuario de las unidades de salud bucodental del Servicio Gallego de Salud. Cad. Aten Primaria. 2007; 14: 168-170.

[17] Soto C, Troya E, Padilla S, Rodríguez R. Morbilidad por urgencias estomatológicas. Clínica Docente Estomatológica 27 de noviembre. Municipio Colón. 2007-2008. Rev Méd Electrón. 2011; 33(1).

[18] López-Silva $M$, Sánchez de Enciso $M$, Rodríguez-Fernández M, Vázquez-Seijas E. Calidad de vida y dolor en atención primaria. Rev. Soc. Esp. Dolor. 2007; 14(1):9-19.

[19] Miotto M, Silotti J, Barcellos L. Dental pain as the motive for absenteeism in a sample of workers. Cien. Saúde Colet. 2012; 17(5):1357-63. DOI:10.1590/S141381232012000500029.

[20] Fodor A, Navarrete E, Caceres E, Muñoz F. Atención de urgencia odontológica en el Hospital Urgencia Asistencia Pública. Rev. Dent. Chile. 2005; 96(3):3-6.

[21] Quezada C, Muñoz D, Cueto A, Barraza S. Reforma de Salud en Chile: Evaluación Garantía Explícita en Salud Oral en Población de 60 Años. Int. J. Odontostomat. 2013; 7 (2): 319-26. DOI:10.4067/S0718381X2013000200024.

[22] Carneiro V, Cavalcanti D, Arruda A, De Andrade R, Lira A et al. Occurrence of Tooth Loss Among Users of the Family Health Strategy in Campina Grande -PB. Revista Brasileira de Ciências da Saúde. 2012; 
$16,137-42$.

DOI:10.4034/RBCS.2012.16.02.04.

[23] Ministerio de Salud (MINSAL). Departamento de Epidemiología, Pontificia Universidad Católica. Departamento de Salud Pública; Universidad Alberto Hurtado. Encuesta Nacional de Salud: Chile 2009-2010. Santiago de Chile, Ministerio de Salud. 2010.

[24] Monsalves M. La odontología que vivimos y no la que queremos; una crítica del escenario actual. Rev. Chil Salud Pública. 2012; 16(2): 241-6. DOI: 10.5354/07173652.2012.23130.

[25] Tonetti MS, Claffey N. Advances in the progression of periodontitis and proposal of definitions of a periodontitis case and disease progression for use in risk factor research. $\mathrm{J}$ Clin Periodontol. 2005; 32 (6):205-8.

[26] World Health Organization. Education for health : a manual on health education in primary health care. 1989.

[27] Contreras A. La promoción de la salud general y la salud oral: una estrategia conjunta. Rev. Clin. Periodoncia Implantol.

\section{CÓMO CITAR ESTE ARTÍCULO}

Cárdenas C, Triviño I, Velásquez $\mathrm{N}$. Caracterización de la Atención del Servicio de Urgencia Dental CESFAM Dr. Jorge Sabat, Valdivia. Appli Sci Dent. 2021:2(2); 515. DOI: 10.22370/asd.2021.2.2.2487
Rehabil. Oral. 2016; 9 (2): 193-202. DOI:10.1016/j.piro.2016.07.003.

[28] Vega J, Solar O, Irwin A, Hernández S, Sandoval $\mathrm{H}$, et al. Determinantes Sociales de la Salud en Chile. En la Perspectiva de la Equidad. 1a edición. Chile. 2005. p. 43.

[29] Instituto Nacional de Estadística (INE). Definiciones estadísticas: Características de la población según edad y sexo. 2020.

Applied Sciences in Dentistry, revista científica de la Facultad de Odontología de la Universidad de Valparaíso, de Acceso Abierto y de Publicación Continua.

Son aceptados trabajos originales e inéditos, abarcando cartas al editor, comunicaciones breves, artículos de investigación, casos clínicos y revisiones bibliográficas.

Correo electrónico de contacto:

contacto.asdj@uv.cl

editor.asdj@uv.cl

Página Web:

https://revistas.uv.cl/index.php/asid

Redes Sociales

Instagram @asd.journal

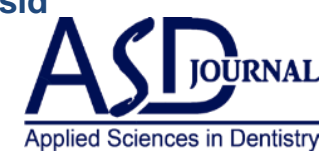

